\title{
Minimally Invasive Technique In Correcting Crooked Nose
}

\author{
Dr Viren Shirish Patil ${ }^{1}$, Dr Shandilya Ramanojam ${ }^{2}$, Dr Sanjay Chandan ${ }^{3}$, \\ Dr Kalyani Gelada ${ }^{4}$, Dr Sanjana Sethi ${ }^{5}$ \\ 1,2,3,4,5 (Department of Oral and maxillofacial Surgery, Bharati Vidyapeeth Dental College and Hospital, India,
} Pune)

\begin{abstract}
As per as facial attractiveness is concerned facial symmetry has a prime importance. Nasal osteotomy also known as rhinoplasty is an aesthetic procedure a surgeon can perform to enhance patient's appearance and give them a new acceptable and aesthetic look. This procedure can be done in the patients have facial asymmetry or patients who are willing to have a new look are the indications due to various reasons such as traumatic injuries, genetic abnormalities etc. Crooked nose has always been a surgical challenge for surgeons. It is of cardinal importance to achieve both functional and esthetic improvements. Deformities of the deviated nose can differ from patient to patient, and that there is no one method that can be used for every deviated nose. Correction requires a complete understanding of the diagnosis and the treatment plan of the patient having nasal deviation. So it is very necessary to a maxillofacial surgeon or a plastic surgeon to have a proper knowledge of surgical management and correction of the unaesthetic look of the patient in a less invasive way.
\end{abstract}

Keywords: esthetic surgery, Crooked nose, Facial asymmetry, Nasal trauma

\section{Introduction}

Facial symmetry is typically considered highly correlated with facial attractiveness. Therefore, it is not surprising that facial deformities that diminish facial symmetry are concerning to patients, who often request reconstructive procedures to restore symmetry. Nasal osteotomy is an aesthetic procedure a surgeon can perform to enhance patient's appearance and give them a new acceptable and aesthetic look.

Due to the nasal deformity patients may face the consequences in both functional and aesthetic terms that cannot be hidden. The nasal axis deviations are classified into three categories; linear (I-shaped), C-shaped, or S-shaped. In the $\mathrm{C}$-shaped crooked nose one side of the dorsum is concave, and the other side is convex. The dorsum and tip in an I-shaped crooked nose (linear) are shifted to one side of the vertical midline of the face. ${ }^{1}$

There are two forces exerted due to trauma which are extrinsic and intrinsic forces. The extrinsic forces are those exerted on the septum by deviated nasal bones, upper lateral cartilages, and connections with the vomer, ethmoid and maxillary crest. The intrinsic forces can be the result of imperfect growth of the septal cartilage or from trauma altering the tissue ultrastructure, after which the deviated cartilaginous tissue always retains an inherent tendency to revert to its initial position. It is important to pin-point the causes of nasal deviation and correct them. Deviated nose is often related to nasal trauma. ${ }^{2}$

The application of anthropometric and craniofacial measures is very important to have a proper surgical results. There should be a precise analysis of the crooked nose which will finally aid in determining the management strategy. However, prior to addressing the nose, facial asymmetries must be elucidated and considered.This case report reveals and emphasize a less-invasive technique in the field of Oral and Maxillofacial surgery as per aesthetics of the face is concerned.

\section{Report Of A Case}

A 21 year-old male patient with a presented to us with a complaint of deviation over the nose and complained about an unaesthetic facial appearance and a deviated nose i.e. a crooked nose. Patient was only concerned about the deviated nose and was not willing to correct the nasal tip deformity and the alar widening. Patient had an allied history of trauma due falling of seasons ball on his nose while catching in a cricket practice session six months ago. At the time of traumatic impact patient gave history of swelling and tenderness over the nose, he then took the primary treatment from the local hospital. Following which there was no history of loss of consciousness, vomiting, oral, convulsion, event amnesia etc. There was history of nasal bleed. Vitals of the patient were stable and the patient was well oriented.

Investigations were advised, CT scan revealed that there was deviation of the dorsum of the nose on the left side. There was malunion of comminuted fracture of the lateral nasal walls and the nasal septum. The patient was admitted in the ward and all routine blood and urine investigations were done and the results were within normal limits. Chest X-ray was advised and there were no any abnormalities. Pre- anaesthetic checkup was done by. A vertical line from the glabella to the gnathion was drawn. This revealed that there was a 
deviation of the nasal septum on the left side. (Fig 1) Patient was then taken up for the procedure in operation theatre. Patient was intubated orally and general anaesthesia was induced. Painting and draping was done (Fig. 3 ). Three stab incisions were taken. Two on the lateral wall of the nose and one on the dorsal hump of the nose was done. The nasal bone was explored and the osteotomy was performed (Fig 2) as a result the deviation of the nasal septum was corrected and the incision site was approximated by 4 ' 0 ' Ethylon suture material (Fig. 4). Patient was then extubated uneventfully. Nasal splint for three weeks was given to the patient.

\section{Figures and Tables}
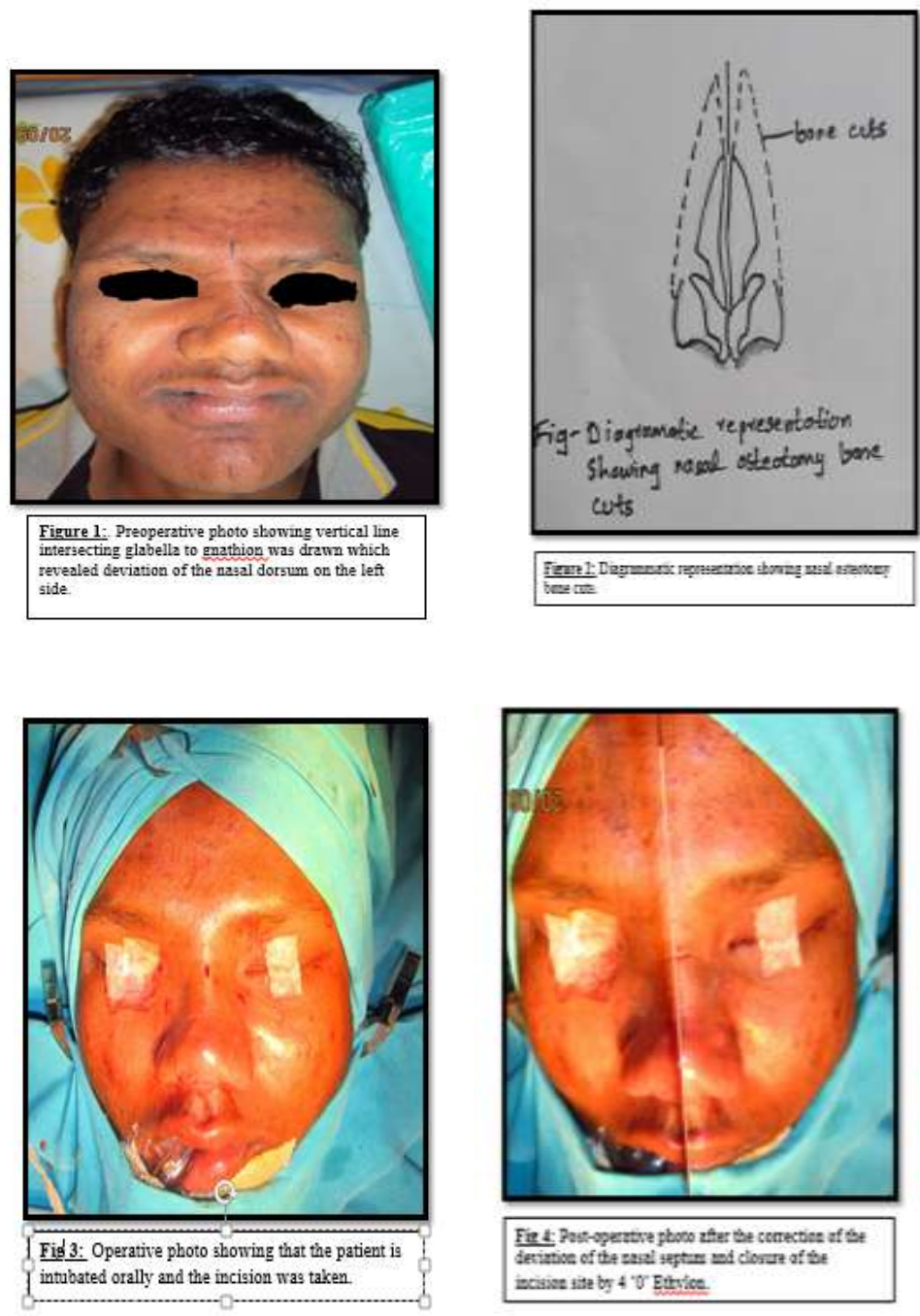

\section{Discussion}

Now-a-days due to increase in the aesthetic awareness in general population, it is necessary for maxillofacial surgeon to have a deep knowledge about the less invasive techniques and methods to correct the facial anomalies which may be due to genetics or due to trauma which indeed will enhance the facial appearance of the patient. The crooked nose is a difficult challenge for surgeons. New surgical techniques has made it possible to make use of procedures that are more extensive than in the past, and are more capable of eliminating the most common complications, such as structural weakening, failure to correct dorsal deviation and relapse. ${ }^{3}$

The ideal technique for lateral nasal osteotomy should maintain the integrity of the nasal mucosa and the periosteal attachment of the bony lateral wall of the nose. The method should be reproducible, safe, precise and do not cause irregularities of the lateral nasal wall. ${ }^{4}$ It is essential that surgeons must detect and discuss the presence of preexisting facial asymmetry which important for both patient education and surgical planning. ${ }^{5}$ The judicious use of anthropometric measurements and esthetic standards, together with the surgeon's perception, may be useful in the evaluation of facial asymmetry. The surgeon must be cognizant of the patient's esthetic expectations and if the expected results are unlikely to meet these expectations the surgeon should not perform the procedure. 


\section{Conclusion}

For these reasons the present case report reveals the therapeutic management based on a specific anatomical and quality of life analysis which will indeed help the facial esthetic surgeon and patient for the betterment of the patient's treatment.

\section{References}

[1]. W.K.A. Hussein et al; Crooked nose: The asymmetric face; Egyptian Journal of Ear, Nose, Throat and Allied Sciences (2015) 16, $237-242$

[2]. W.K.A. Hussein; Crooked nose: An update of management strategies; Egyptian Journal of Ear, Nose, Throat and Allied Sciences (2015) 16, 1-7

[3]. Tardy Jr ME, Becker D, Weinberger M. Illusions in rhinoplasty. Facial Plast Surg. 1995; 11:117-137.

[4]. Ghassemi et al; Lateral nasal osteotomy: a comparative study between the use of osteotome and a diamond surgical burr - a cadaver study; Head \& Face Medicine 2013, 9:41

[5]. Chatrath P, De Cordova J, Nouraei SA, Ahmed J, Saleh HA. Objective assessment of facial asymmetry in rhinoplasty patients. Arch Facial Plast Surg. 2007; 9:184-187. 